\title{
Development of an airline model with non-uniform parameters for coordination studies of isolation
}

\author{
Edgar Alejandro Uribe Guer ${ }^{1}$, Germán Moreno O. ${ }^{2}$
}

\author{
${ }^{1}$ Empresas Públicas de Medellín (EPM) - Medellín, Colômbia. \\ ${ }^{2}$ Universidad de Antioquia (UdeA) - Cl. 67 \#53 - 108, Medellín, Antioquia, Colômbia. \\ Email: edgar.uribe@epm.com.co,gmoreno@udea.edu.co
}

Received: November $10^{\text {th }}, 2017$.

Accepted: January $15^{\text {th }}, 2018$.

Published: March $30^{\text {th }}, 2018$.

Copyright (C2016 by authors and Institute of Technology Galileo of Amazon (ITEGAM).

This work is licensed under the Creative Commons Attribution International

License (CC BY 4.0).

http://creativecommons.org/licenses/by/4.0/ (c) (1) (3)(2) Open Actes:

\begin{abstract}
The modeling of the corona effect, the stresses induced by indirect atmospheric discharges and the non-uniformity of parameters in transmission lines have been modeled efficiently, usually separately, in studies that involve knowledge of the response of lines to transient disturbances. In this article, we present a line model that includes these phenomena simultaneously and with a high degree of precision. The dependence on the frequency of the parameters of the transmission line was considered. The consolidated model makes use of the wide variety of simulation structures available today, appropriately identifying the critical aspects in each of the phenomena to be evaluated. Sensitivity analyzes were carried out and evaluations of the main characteristics of each of the phenomena with the aim of validating the responses obtained before a set of selected disturbances. Good concordance was observed between the answers obtained and the expected theoretical answers.
\end{abstract}

Keywords: Corona effect, Indirect atmospheric discharges, transmission lines.

\section{Desarrollo de un modelo de línea aérea con parámetros no uniformes para estudios de coordinación de aislamiento}

\begin{abstract}
RESUMEN
El modelamiento del efecto corona, las tensiones inducidas por descargas atmosféricas indirectas y la no uniformidad de parámetros en líneas de transmisión han sido modeladas de forma eficiente, usualmente de forma separada, en estudios que involucran el conocimiento de la respuesta de líneas ante perturbaciones transitorias. En el presente artículo, se presenta un modelo de línea que incluye estos fenómenos de forma simultánea y con un alto grado de precisión. La dependencia con la frecuencia de los parámetros de la línea de transmisión fue considerada. El modelo consolidado hace uso de la amplia variedad de estructuras de simulación disponibles en la actualidad, identificando apropiadamente los aspectos críticos en cada uno de los fenómenos a evaluar. Análisis de sensibilidad fueron llevados a cabo y evaluaciones de las principales características de cada uno de los fenómenos con el ánimo de validar las respuestas obtenidas ante un conjunto de perturbaciones seleccionadas. Buena concordancia fue observada entre las respuestas obtenidas y las respuestas teóricas esperadas.
\end{abstract}

Palabras Claves: Efecto corona, Descargas atmosféricas indirectas, Línea de transmisión.

\section{INTRODUCCIÓN}

Los incrementos en las capacidades de procesamiento de los equipos empleados para el cálculo de transitorios electromagnéticos y la disponibilidad de modelos más precisos de los diferentes fenómenos de interés en un estudio de coordinación de aislamiento, han desencadenado la creación de estructuras de simulación para elementos como líneas, aisladores, generadores, etc, mucho más complejas pero también más ajustadas a la realidad, y por consiguiente más confiables.

La forma habitual de modelar una línea de transmisión está basada en una serie de suposiciones o simplificaciones de la 
disposición física y el comportamiento estático y dinámico de la línea bajo análisis. La revisión del modelo empleado para el efecto corona, la no uniformidad de parámetros de propagación y la inclusión del desempeño de la línea ante tensiones inducidas ocasionadas por descargas atmosféricas, es el núcleo del presente artículo.

En las primeras secciones del documento se abordan los diferentes modelos de los tres fenómenos analizados. En las siguientes secciones se evalúa la sensibilidad de la respuesta del modelo de línea construido ante variaciones de los parámetros internos del modelo, y ante alteraciones en la amplitud y la frecuencia de las perturbaciones consideradas. Además se efectúan los ajustes necesarios en cuanto a adecuación de las condiciones de simulación de los tres fenómenos de forma simultánea.

Los resultados obtenidos y los tiempos de procesamientos de información tomados por el modelo de línea para las simulaciones llevadas a cabo en relación con modelos típicos de línea, permiten evaluar bajo qué condiciones es apropiado emplear el modelo construido o cuando es mejor emplear las estructuras habituales de modelamiento.

\section{MODELAMIENTO DEL EFECTO CORONA EN LÍNEAS DE TRANSMISIÓN AÉREAS}

El efecto corona cuenta con un variado conjunto de modelos [2] que permiten introducir el fenómeno apropiadamente en simulaciones que involucran la respuesta de una línea de transmisión. Sin embargo, todos los modelos disponibles tienen como objetivo primordial representar la característica v-q (ver Figura 1) de los conductores que componen la línea.

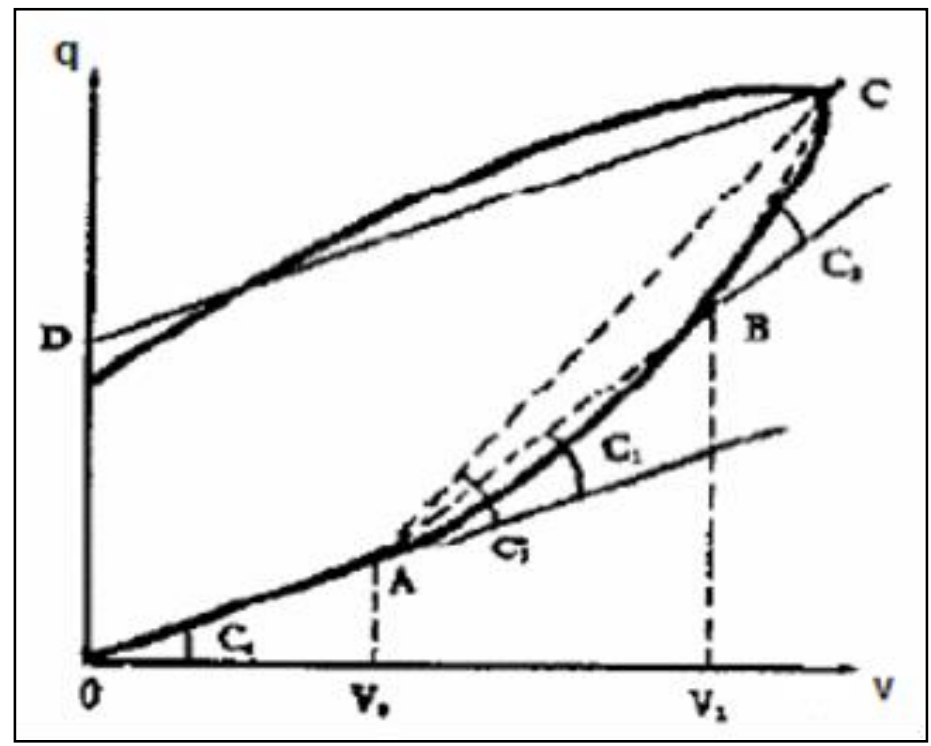

Figura 1: Curva v-q.

Fuente: Los Autores, (2017).

$\mathrm{C}_{0}=$ Capacitancia Geométrica de la línea. $\mathrm{V}_{0}=$ Voltaje de Ignición del Efecto Corona. $\mathrm{C}_{1}, \mathrm{C}_{2}, \mathrm{C}_{1}{ }^{\prime}=$ Capacitancias incrementales. 0, A, B, C, D= Puntos de control de ajuste de la característica v-q.

Los dos grandes grupos de modelos empleados son los que reconstruyen la curva a partir de equivalentes analógicos circuitales, representando la presencia del efecto corona mediante elementos como resistencias, capacitancias, fuentes, diodos, entre otros elementos circuitales típicos; y aquellos que consideran el efecto mediante elementos no lineales calculados a partir de la solución completa de la red en el punto donde ha sido concentrado el efecto de forma distribuida. El principal inconveniente que se ha encontrado en el uso de los equivalentes circuitales del efecto corona son las oscilaciones numéricas propias del swicheo de los diodos que se emplean en las ramas que modelan la aparición del fenómeno como tal. En ambos casos, las curvas v-q son aproximadas mediante tramos de línea recta y ajustadas para la característica experimental obtenida del desempeño de los conductores en pruebas de laboratorio [3]. Mediante una serie de suposiciones físicas [2,3,4] propias del fenómeno, es posible calcular los parámetros de líneas reales a partir de los resultados obtenidos mediante pruebas de laboratorio. Usando estas técnicas fueron calculados los parámetros de ajuste para el efecto corona considerado en la línea bajo estudio seleccionada (Figura 1). Para cada tramo, con una geometría de línea particular y por consiguiente una capacitancia diferente, se efectúa el ajuste de la capacitancia obtenida [2,3], logrando de esta forma la construcción de un puente directo entre el modelamiento del efecto corona de una forma semidistribuida y la metodología de modelamiento de líneas no uniformes adoptada.

Las simulaciones efectuadas fueron llevadas a cabo mediante el uso del programa ATP. El método de compensación incluido en dicho software facilitó la obtención de los equivalentes de Thévenin en los diferentes puntos a lo largo de la red en los que se concentró el efecto corona y donde fueron conectadas las ramas correspondientes. La longitud de los tramos de línea entre los que deben ubicarse las ramas corona es de alrededor de unos 50 ó 100 m [2]. Esta selección sugerida, es independiente del tipo de perturbación a la cual se someta la línea. En general, la respuesta exhibida para tramos con longitudes menores de $50 \mathrm{~m}$ no mostró variaciones apreciables para una sobretensión del tipo rayo [2] en una línea con características similares. Para excitaciones más lentas o de menor frecuencia equivalente se pueden esperar valores de longitud mínima de tramo superiores para los cuales no se observen variaciones considerables en las respuestas de la línea en presencia del corona. Se ilustra en la Figura 2 el esquema circuital de ATP empleado:

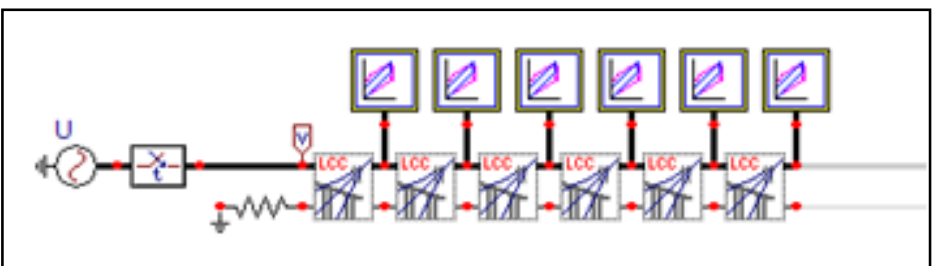

Figura 2. Equivalente Circuital empleado en la representación del efecto corona.

Fuente: Los Autores, (2017).

\section{MODELAMIENTO DE TENSIONES INDUCIDAS POR DESCARGAS ELÉCTRICAS ATMOSFÉRICAS INDIRECTAS EN LÍNEAS DE TRANSMISIÓN AÉREAS}

Se seleccionó el modelo expuesto en [1] por la forma como introducen las tensiones inducidas en la línea, a partir de dos fuentes en los extremos o apoyos más cercanos del punto de impacto de la descarga como se muestra en la Figura 3. Bajo esa concepción, se tiene que la magnitud de las tensiones inducidas en los extremos de una línea será independiente del número de tramos que sean considerados para representar la no uniformidad de la línea; el efecto neto de la variación del número de tramos de línea se verá reflejado sobre las características de propagación de las tensiones inyectadas en los extremos de la línea. 


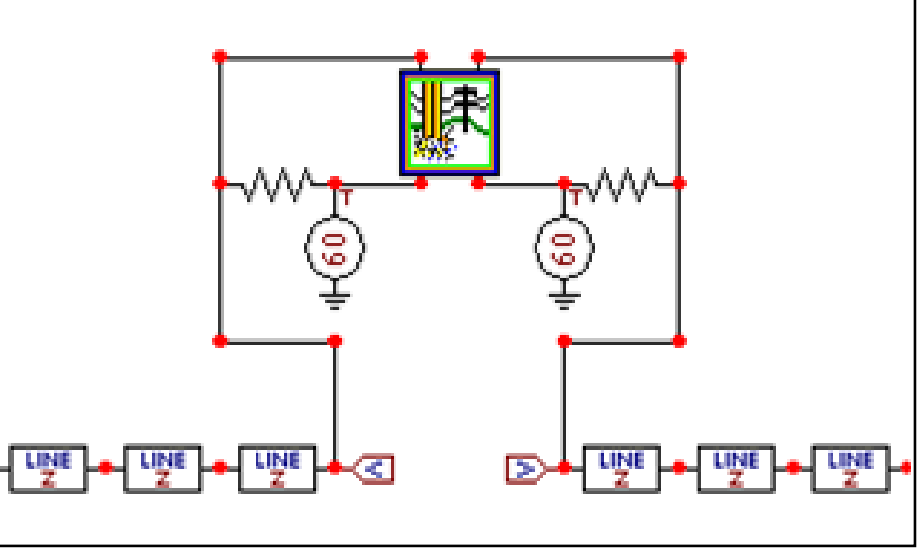

Figura 3: Equivalente circuital modelo de tensiones inducidas por descargas indirectas.

Fuente: Los Autores, (2017).

\section{NO UNIFORMIDAD EN LOS PARÁMETROS DE PROPAGACIÓN DE UNA LÍNEA DE TRANSMISIÓN AÉREA}

La no uniformidad en una línea de transmisión es algo propio de su naturaleza física. Las irregularidades del terreno, la existencia de vanos prolongados, las variaciones en las condiciones atmosféricas, etc son factores que afectan enormemente las características de propagación de una línea de transmisión. Los grandes avances que se han alcanzado en torno al modelamiento de líneas de transmisión uniformes, en cuanto a precisión y a tiempo de cálculo, permiten pensar que la mejor forma de incluir la no uniformidad de una línea de transmisión o de un tramo de ésta, es mediante una colección finita de líneas de transmisión uniformes [5,6].

El problema se convierte entonces en una colección finita de reflexiones y transmisiones de onda viajera, en las cuales los coeficientes de reflexión y transmisión son dependientes de la posición y de la geometría del tramo de línea. La solución de este problema es equivalente a ubicar una serie de tramos de línea en cascada con parámetros físicos o eléctricos diferentes. La dependencia de la frecuencia se obtiene como resultado del efecto acumulado de todos los tramos uniformes, cada uno de los cuales es dependiente de la frecuencia. Tal fue la solución que se empleó y de la cual se muestra en la Figura 4 la forma como fue simulado:

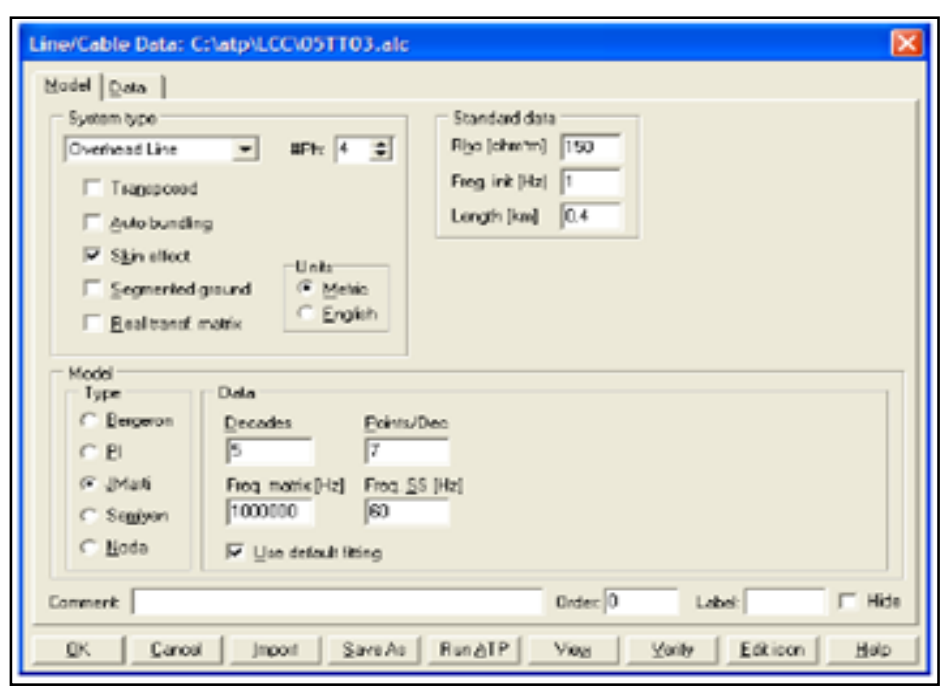

(a)

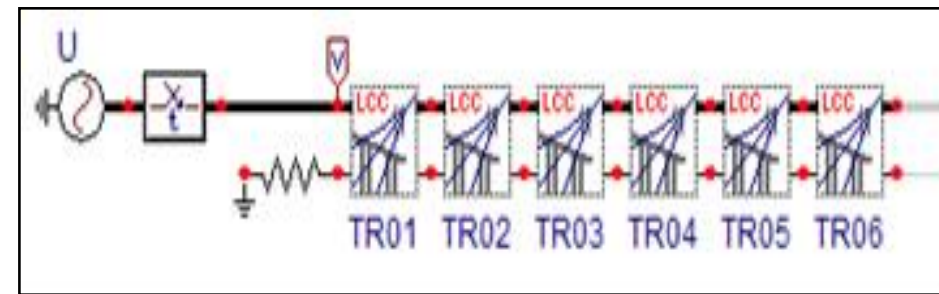

(b)

Figura 4: (a) Detalle del ajuste empleado en al ATP para un tramo de línea. (b) Equivalente Circuital de una línea no uniforme representada por 6 tramos de línea en cascada, de parámetros uniformes.

Fuente: Los Autores, (2017).

En general, las variaciones en la tensión a lo largo de un circuito similar al mostrado en la figura 4 , variando el número de tramos pero manteniendo la longitud total, y ajustando las posiciones como las coordenadas promedio en el tramo representado, no fueron muy apreciables.

Se percibieron variaciones mínimas en cuanto a magnitud y de atraso en las señales observadas el final de la línea. Esta observación permite concluir que, al menos para una corriente de excitación tipo rayo, maniobra o de estado estacionario, como las incluidas en el presente análisis, no es relevante modelar una línea como uniforme o como no uniforme ya que las respuestas dinámicas y de estado estacionario ante perturbaciones de tipo transitorio no son muy significativas. Es importante señalar que lo expuesto con anterioridad es válido, siempre y cuando, se evalúe el desempeño en líneas cuya longitud total sea la misma, y en las cuales se adopten un número diferente de tramos uniformes de línea independiente ante una y sólo una perturbación similar a las estudiadas, para efectos de comparación de las respuestas. Sin embargo, como se muestra en la Figura5, una misma línea responderá de una forma coherente con la frecuencia del tipo de perturbación a la cuál es sometida, lo que se verifico fue el efecto que sobre dicha respuesta tuviera considera mas o menos tramos.

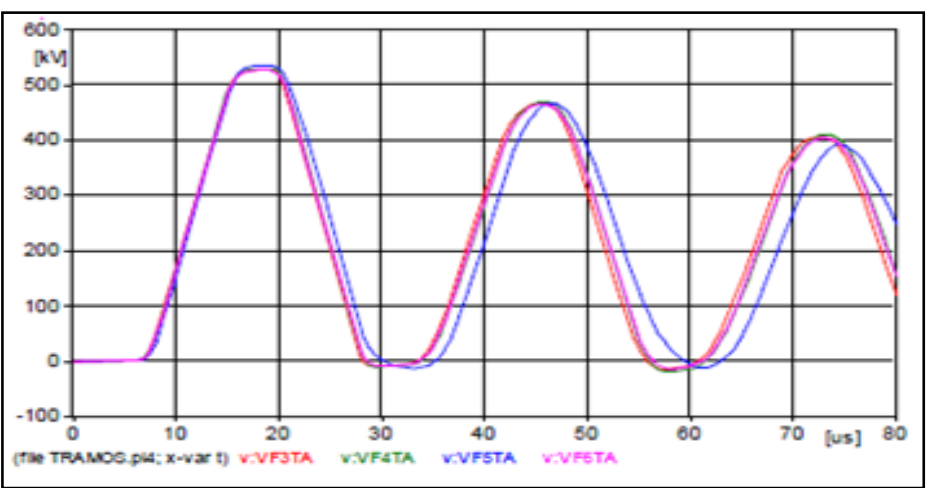

(a)

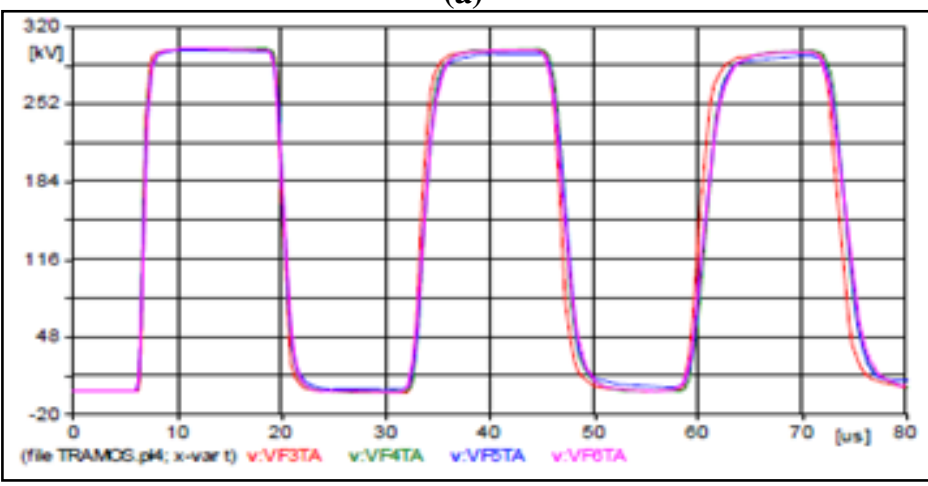

(b) 


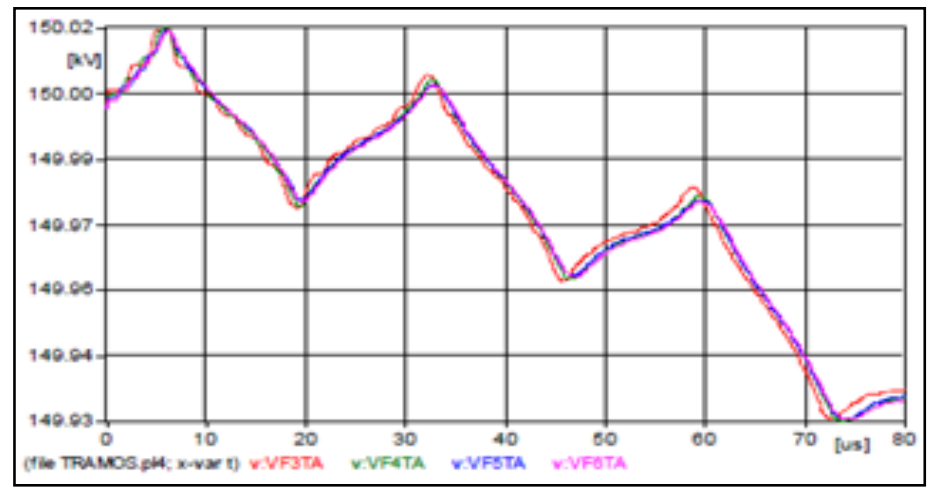

(c)

Figura 5: Respuesta al final de la línea cuando es modelada mediante 3, 4, 5 y 6 tramos (VF3TA, VF4TA, VF5TA y VF6TA, respectivamente). (a) Perturbación Tipo descarga atmosférica. (b) Perturbación tipo maniobra. (c) Detalle de la respuesta a frecuencia industrial $(60 \mathrm{~Hz})$.

Fuente: Los Autores, (2017).

\section{MODELO DE LÍNEA AÉREA CONSOLIDADO}

Por modelo consolidado se entiende la estructura de simulación que incluye las tres características analizadas en el presente artículo. La línea que fue considerada se asimila a una salida de un pórtico de una subestación a una estructura de transmisión en tendido horizontal. La distribución analizada se muestra en la Figura 6:

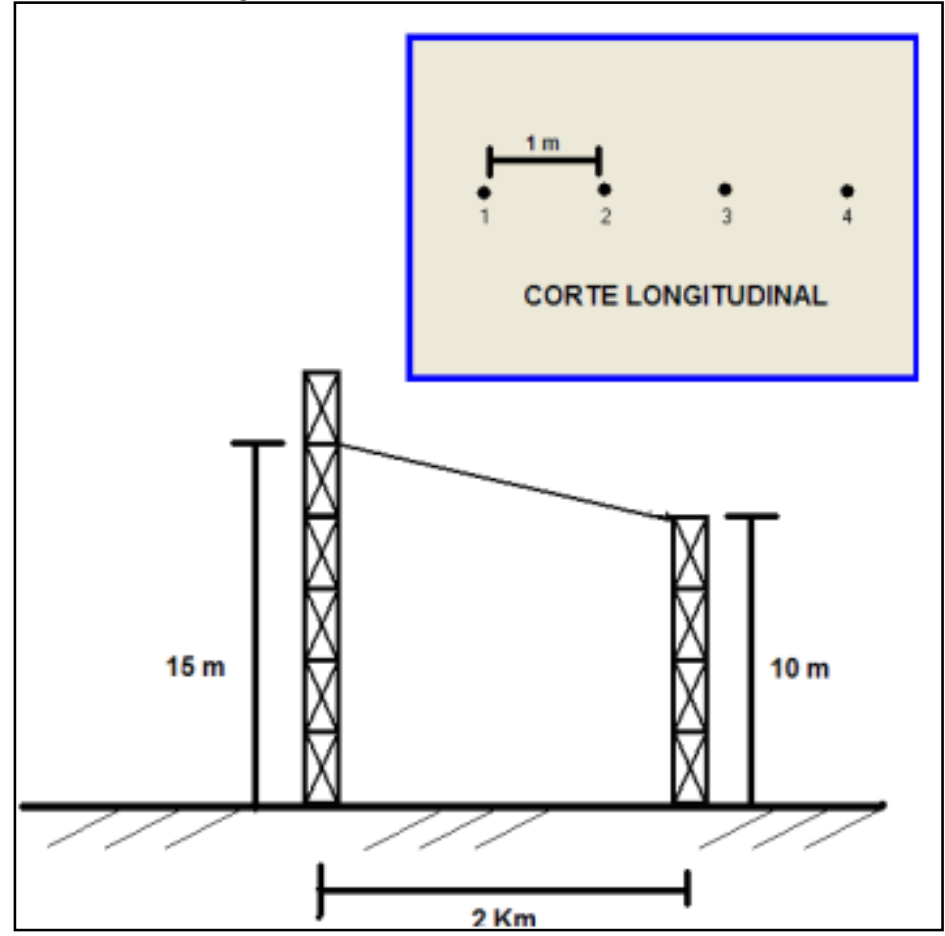

Figura 6: Afloramiento de línea.

Fuente: Los Autores, (2017).

Fue seleccionada de tal forma que fuera un tramo particularmente no lineal en cuanto a la variación de la altura de cada uno de los conductores a medida que se avanza de forma longitudinal en la línea. Teniendo en cuenta que tanto para el modelamiento del efecto corona, como para considerar la no linealidad de la línea deben considerarse tramos en serie, es importante conocer cuál es el número de tramos apropiados para considerar ambos fenómenos. Esto sin sacrificar la precisión de los resultados o incrementar demasiado el tiempo de cálculo de las respuestas del modelo.
Para el caso del efecto corona, como ya se había señalado con anterioridad, se tiene que según [2] a partir de tramos de línea de $50 \mathrm{~m}$ no se aprecian variaciones significativas en las respuestas obtenidas. Por tal razón se varió el número de tramos que componían la línea hasta que ninguna variación significativa fuera observada en las tensiones a lo largo de la línea. De esta forma fue posible evaluar la concordancia entre el modelo construido y la respuesta esperada según [2].

Un aspecto de vital importancia en el proceso de consolidación del modelo, es la selección del tamaño de paso $\left(\Delta_{\mathrm{t}}\right)$ y del tiempo total de la simulación $\left(\mathrm{T}_{\max }\right)$. Debe asegurarse que dichos tiempos sean congruentes con las constantes de propagación de los tramos de línea que se consideren para efectos de la no linealidad, así como con los algoritmos internos de alguno de los modelos tenidos en cuenta, en los cuales pueda ser importante elegir un tamaño de paso en particular. Para nuestro caso, se eligió un tiempo máximo en cada simulación de por lo menos 4 veces el tiempo de viaje total de una perturbación que atravesara la totalidad de la línea viajando a la velocidad de la luz en el vacío (c0). Sin embargo, es importante señalar que las velocidades de propagación obtenidas para los tramos de línea estuvieron siempre por debajo de la referencia empleada para la selección del tamaño máximo, como era de esperarse. De esta forma, es posible tener presentes todas las reflexiones que afectan en mayor medida el desempeño de la línea y compensar los pequeños retrasos asociados con el efecto corona sobre las perturbaciones que sobrepasen la tensión de ignición del fenómeno, ya que como es característico, además de presentarse una atenuación en las señales de tensión que se propagan bajo la presencia del fenómeno, también se esperaría, debido a la ionización en torno a los conductores, un efecto de "frenado" sobre las ondas de tensión en tránsito. Con el fin de disminuir el tamaño de las reflexiones de onda en los extremos de la línea se incluyeron impedancias cercanas a las impedancias típicas de los circuitos de transmisión analizados, al final del arreglo de los tramos dispuestos en serie. De igual forma, el tiempo total de simulación puede variarse en cada caso dependiendo las necesidades como en el caso de las tensiones inducidas por descargas indirectas en las cuales debido a dinámicas muchísimo más rápidas y locales pueden seleccionarse tiempos totales mayores, como se muestra al final de la presente sección.

Para la selección del tamaño de paso de la simulación es necesario considerar que mientras más pequeño sea el tamaño seleccionado, mucho más tiempo tomará la simulación. Además de que incluir un mayor número de tramos de línea también acarreará mayores tiempos de cálculo. En el caso del efecto corona, es importante señalar que la inclusión del fenómeno se hace por tensión y no por tiempo, y que la dependencia con la frecuencia del fenómeno fue considerada al momento de ajustar las diversas características v-q obtenidas en $[2,4]$ a la geometría de la línea, mediante la metodología sugerida en [4]. En el caso de las tensiones inducidas, el modelo seleccionado cuenta con un tamaño de paso y un número máximo de pasos a considerar propios de la rutina de inyección de la tensión inducida en cada una de los extremos; para este caso el tamaño de paso se eligió igual al empleado para el resto de la simulación y el número máximo de pasos, se escogió de tal modo que la ventana de tiempo que generara siempre fuese menor que el tiempo máximo total seleccionado para la simulación completa de la respuesta de la línea. Cabe señalar que fue el modelo de las tensiones inducidas el que impuso el tamaño de paso requerido. La razón fundamental de esto, es que el modelo de tensiones inducidas considera la respuesta particular de un tramo de línea mediante un acercamiento a la condición de acople entre el canal de la 
descarga vertical del rayo y un tramo de línea en particular, de longitud comparable a la distancia hasta el punto de impacto del rayo; de esta forma, se requieren tramos de línea que sean apropiados para distancias mínimas de impacto de unos 30 ó $50 \mathrm{~m}$ al eje de la línea.

A pesar de que podría elegirse un tamaño de paso específico para transitorios del tipo rayo, maniobra o frecuencia industrial, se tomaron los tamaños de paso y los tiempos máximos impuestos por el modelo de tensiones inducidas, con el ánimo de capturar en cualquier tipo de simulación dinámicas de muy corta duración, así como permitir un nivel de detalle superior de las no linealidades de la línea, a través de la admisión de tramos de línea de hasta unos 15 ó 20 m, valores muy por debajo de los mínimos de longitud de los tramos sugeridos para el modelamiento del efecto corona y para un nivel de detalle apropiado para efectos de modelar las no linealidades de la línea. A continuación puede verse la respuesta transitoria ante la misma perturbación tipo rayo ( $90 \mathrm{kV}, 8 \mu \mathrm{s}$ tiempo de frente y $120 \mu \mathrm{s}$ tiempo de cola), en la fase A del extremo de una línea que fue modelada mediante 3,4,5 y 6 tramos (VF3TA, VF4TA, VF5TA y VF6TA, respectivamente). Las tensiones obtenidas se muestran a continuación (Figura 7).

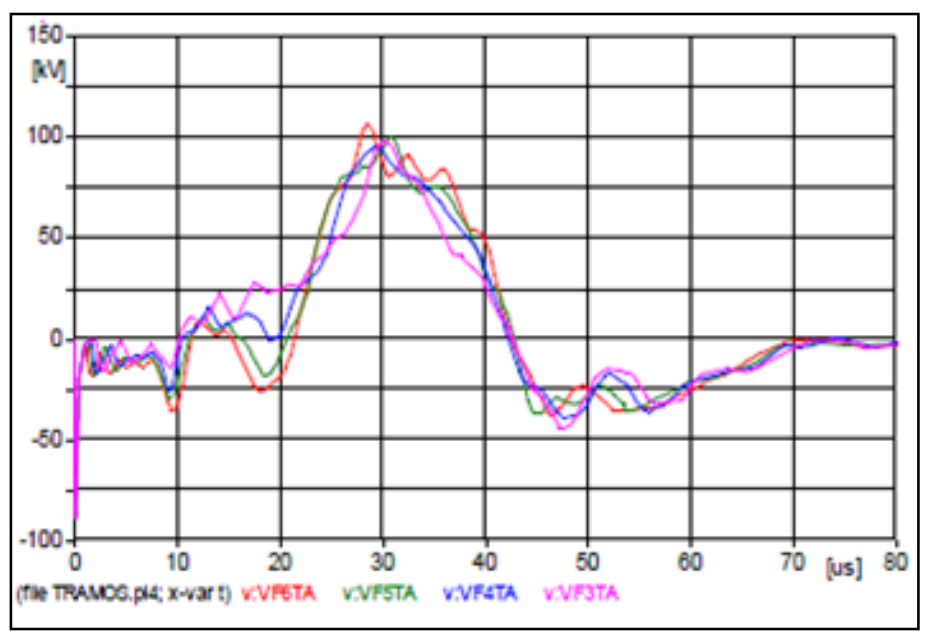

(a)

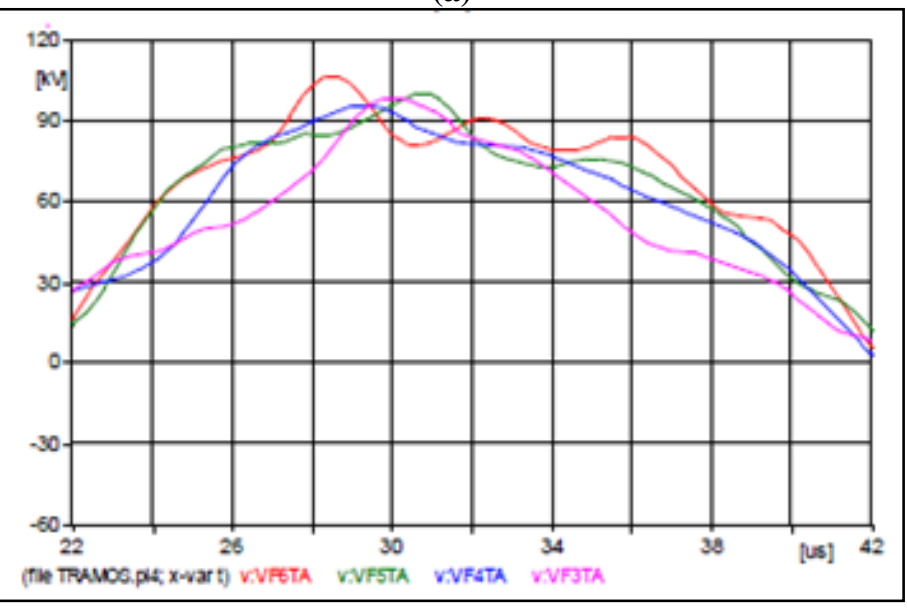

(b)

Figura 7: Detalle de la respuesta del modelo consolidado de línea ante una perturbación tipo descarga atmosférica, en la fase A del extremo de una línea que fue modelada mediante 3,4,5 y 6 tramos (VF3TA, VF4TA, VF5TA y VF6TA, respectivamente). (a) Respuesta completa $(0-80 \mu \mathrm{s})$. (b) Detalle en el pico de tensión obtenido (22-42 $\mu \mathrm{s})$

Fuente: Los Autores, (2017).

Las tensiones alcanzadas con los modelos donde fueron incluidos más tramos son mayores. De la fig6, se puede observar una diferencia de unos $15 \mathrm{kV}$ entre las tensiones al final de la línea para el modelamiento con 6 y 5 tramos. De esta manera se justifica entonces el uso de un mayor número de tramos, para afinar algunos ejercicios de modelamiento en los cuales se requiera de un nivel de precisión especial; sin embargo, para la mayoría de las simulaciones bastaría con un pequeño número de tramos debido a que los errores en las respuestas podrían ser compensados con los factores de seguridad empleados en la definición de los niveles de protección de los equipos. De todas formas, el mayor consumo de tiempos de cálculo y el sobredimensionamiento habitual en sistemas de aislamiento eléctrico, sugieren el uso de modelos de línea uniformes ajustadas a cada problema en particular. La construcción de dichos criterios de decisión se sustenta en el uso de modelos ampliamente comprobados para cada uno de los fenómenos empleados y en la adopción de un criterio que permita obtener respuestas rápidas sin sacrificar precisión.

Cabe señalar que el modelo de cada tramo dependiente de la frecuencia fue seleccionado de manera que el número de polos fuera un poco mayor que el recomendado usualmente [6,7] para el rango de frecuencias seleccionado. Esto con el fin de garantizar que las respuestas en frecuencia requeridas de los tramos de línea, representen fielmente el comportamiento transitorio en el rango de frecuencia estudiado (1-1000 kHz) sin exhibir oscilaciones numéricas o algún inconveniente asociado a la etapa de ajuste.

Para el caso de las tensiones inducidas, se eligió en cada caso el tramo más cercano al punto de impacto de la descarga indirecta y en ese sitio fueron inyectadas las perturbaciones en forma de fuentes de tensión en los dos apoyos más cercanos. Se probó la respuesta exhibida para efectos de verificación en la misma línea cuando era modelada mediante tres tramos. En la Fig8 se exhibe la tensión inducida en cada uno de los extremos de la línea no uniforme. El tamaño de paso y el tiempo máximo empleados en la simulación fueron $2 \mathrm{e}^{-9} \mathrm{~s}$ y $5 \mathrm{e}^{-7} \mathrm{~s}$, respectivamente:

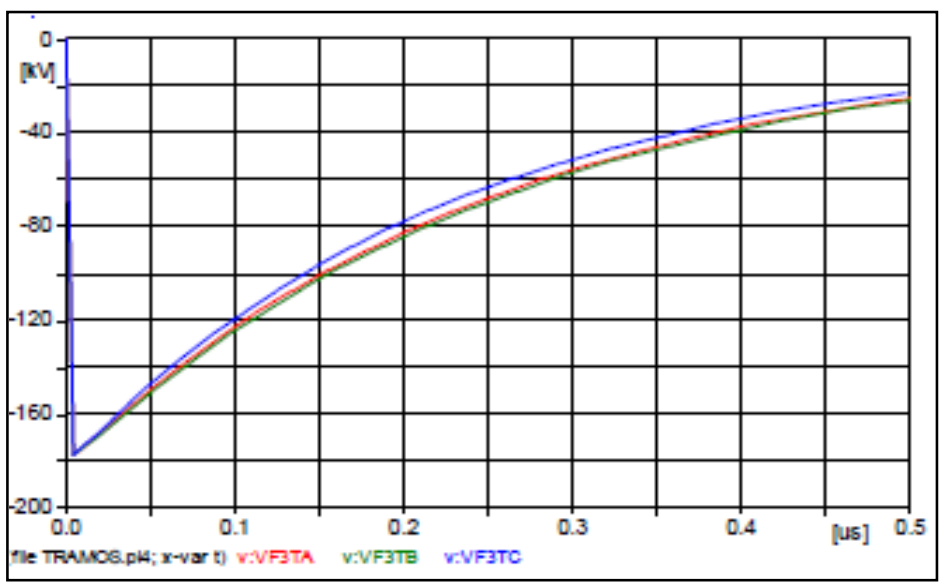

Figura 8: Tensión inducida en una línea no uniforme modelada mediante tres tramos uniformes de igual longitud.

Fuente: Los Autores, (2017).

Es importante señalar que la respuesta exhibida corresponde exclusivamente a la tensión inducida inyectada en los extremos de la línea terminada en un par de impedancias equivalentes para prevenir la reflexión de alguna porción de la onda incidente y evaluar la magnitud de las tensiones obtenidas. La descarga indirecta se modeló impactando en la tierra a $500 \mathrm{~m}$ de la línea, con una corriente de rayo de $35 \mathrm{kA}$, un tiempo de frente de $1,2 \mu \mathrm{s}$ y un tiempo de cola de $50 \mu \mathrm{s}$. Es importante señalar que el modelo de línea construido puede ser empleado para diferentes tipos de perturbaciones y que las tensiones inducidas por descargas indirectas serían sólo un tipo de 
perturbación para la cual fue adaptado el modelo de tal forma que fuera capaz de reproducir un fenómeno del cual no se cuenta con modelos estándar disponibles acoplados a modelos de línea. Por tal razón, podría estar presente o no en alguna simulación, dependiendo del alcance del estudio en el cual se emplee el modelo en el futuro.

\section{CONCLUSIONES}

Tres importantes características del funcionamiento de una línea de transmisión fueron analizadas. Modelos probados del funcionamiento aislado de cada uno de los modelos fueron seleccionados y adicionados a un único modelo de línea que respondiera apropiadamente a las condiciones de simulación seleccionadas. Análisis de sensibilidad fueron llevados a cabo sobre la respuesta exhibida por la línea cuando fue representada como una colección finita de tramos de línea uniformes con parámetros de propagación dependientes de la frecuencia. Se adoptó como criterio de precisión que al incrementar el nivel de detalle de las no uniformidades de la línea no se acarrearan variaciones considerables en las respuestas obtenidas de las simulaciones llevadas a cabo y que no se eliminaran dinámicas propias y particulares de cada uno de los fenómenos considerados.

Contar con modelos de línea uniformes bastante flexibles y precisos, sumado al incremento en las capacidades de cálculo de las herramientas actuales, permite elaborar modelos modulares complejos de líneas de transmisión, como se mostró a lo largo del trabajo. Para cada caso es primordial evaluar el número apropiado de tramos por considerar y ser riguroso en la selección y evaluación de los parámetros de los fenómenos que quieren ser representados al correspondiente nivel de detalle.

\section{REFERENCIAS}

[1] H.K. Hoidalen, "Analytical Formulation of LightningInduced Voltages on Multiconductor Overhead lines Above Lossy Ground", IEEE Tans. On Electromagnetic Compatibility, Vol. 45, No.1, February 2003, pp. 92-100.

[2] J. Sandoval Carneiro, J.R. Marti, "Evaluation of Corona and Line Models in Electromagnetic Transient Simulations", IEEE Trans. On Power Delivery, Vol.6, No.1, January 1991, pp. 334342.

[3] P. Sarma Maruvada, D.H. Nguyen, \& H. Hamadami-Zadeh, "Studies on Modeling Corona Attenuation of Dynamic Overvoltages", IEEE Trans. On Power Delivery, Vol.4, No.2, April 1989, pp. 1441-1449.

[4] M. Mihailescu-Suliciu, I. Suliciu, "A Rare Type Constitutive Equation for the Description of the Corona Effect", IEEE Trans. On Power Apparatus and Systems, Vol.100, No.8, August 1981, pp. 3681-3685.

[5] A.I. Ramirez, A.Semlyen, \& R.Iravani., "Modeling Nonuniform Transmission Lines for Time Domain Simulation of Electromagnetic Transients", IEEE Trans. On Power Delivery, Vol.18, No.3, July 2003, pp. 968-974.

[6] A.Semlyen, "Some Frequency Domain Aspects of Wave Propagation on Nonuniform Lines", IEEE Trans. On Power Delivery, Vol.18, No.1, January 2003, pp. 315-322.
[7] J.R. Marti, "Accurate Modeling Of Frequency-Dependent Transmission Lines in Electromagnetic Transient Simulations", IEEE Trans. On Power Apparatus and Systems, Vol.101, No.1, January 1982, pp. 147-157. 